\title{
PARALLEL TRANSLATION OF CURVATURE ALONG GEODESICS
}

\author{
JAMES J. HEBDA
}

\begin{abstract}
According to the Cartan-Ambrose-Hicks Theorem, two simplyconnected, complete Riemannian manifolds are isometric if, given a certain correspondence between all the broken geodesics emanating from a point in one manifold; and all those emanating from a point in the other, the parallel translates of the curvature tensor agree along corresponding broken geodesics. For generic metrics on a surface, the hypothesis can be refined so that it is enough to compare curvature along corresponding unbroken geodesics in order to obtain the isometry.
\end{abstract}

Introduction. Let $M$ and $\bar{M}$ be two complete, connected Riemannian manifolds of the same dimension. Given points $p \in M$ and $\bar{p} \in \bar{M}$, and a linear isometry $I: T_{p} M \rightarrow T_{\bar{p}} \bar{M}$ between tangent spaces, there corresponds to each geodesic $g:[0,1] \rightarrow M$ emanating from $p$ (i.e. $g(0)=p$ ), a unique geodesic $\bar{g}:[0,1] \rightarrow \bar{M}$ emanating from $\bar{p}$ and satisfying $\bar{g}^{\prime}(0)=I\left(g^{\prime}(0)\right)$ where the prime denotes tangent vector. For each such geodesic $g, I_{g}=P_{\bar{g}} \circ I \circ P_{g}^{-1}$ defines a linear isometry from $T_{g(1)} M$ onto $T_{\bar{g}(1)} \bar{M}$ where $P_{g}$ and $P_{\bar{g}}$ denote parallel translation along $g$ and $\bar{g}$ respectively. Because an isometry preserves curvature and commutes with parallel translation, the existence of an isometric immersion $F: M \rightarrow \bar{M}$ such that $F(p)=\bar{p}$ and $d F=I$ at $p$ would imply the condition:

For every geodesic $g$ emanating from $p$, and for all vectors $X, Y$, $Z \in T_{g(1)} M$,

$$
I_{g}(R(X, Y) Z)=\bar{R}\left(I_{g}(X), I_{g}(Y)\right) I_{g}(Z)
$$

where $R$ and $\bar{R}$ are the respective Riemann curvature tensors of $M$ and $\bar{M}$.

On the other hand, if condition $(*)$ holds, then there exists an isometry $f$ of a neighborhood of $p$ onto a neighborhood of $\bar{p}$ with $d f=I$ at $p$ [4]. Thus, if both $M$ and $\bar{M}$ were real analytic and $M$ simply connected, then $f$ could be extended by analytic continuation to a global isometric immersion $F: M \rightarrow \bar{M}[\mathbf{1 1}]$. Ambrose [1] obtains a global isometric immersion in the smooth $\left(C^{\infty}\right)$ case when $M$ is simply connected by assuming a stronger version of $(*)$ involving singly broken geodesics emanating from $p$. Whether Ambrose's version of $(*)$ must be assumed in order to obtain the global isometric immersion, or whether it is sufficient to assume $(*)$, is

Received by the editors February 10, 1986. The contents of this paper were presented on November 2, 1985, in the special session on Differential Geometry during the 823rd meeting of the AMS held in Columbia, Missouri.

1980 Mathematics Subject Classification (1985 Revision). Primary 53C20; Secondary 34A10. 
an open question $[\mathbf{1}]$. The main result of the present work shows that assuming $(*)$ suffices for "generic" surfaces.

THEOREM 1. Suppose $M$ and $\bar{M}$ are both complete, connected Riemannian manifolds of dimension two, and, given the above situation, that the condition (*) holds. If $M$ is simply connected, and if every compact subset of the cut locus of $p$ has finite 1-dimensional Hausdorff measure, then there is an isometric immersion $F: M \rightarrow \bar{M}$ with $F(p)=\bar{p}$ and $d F=I$ at $p$.

REMARKS. (1) For surfaces $(*)$ takes the particularly simple form: For every geodesic $g$ emanating from $p, K(g(1))=\bar{K}(\bar{g}(1))$ where $K$ and $\bar{K}$ are the Gaussian curvature functions on $M$ and $\bar{M}$ respectively.

(2) If the cut locus in a surface is triangulable, every compact subset has finite Hausdorff 1-measure. The cut locus is triangulable for real analytic metrics $[\mathbf{3}$, 12], and, if $M$ is compact, for all metrics in an open dense subset of the space of Riemannian metrics on $M[\mathbf{1 5}]$.

(3) Are there any complete surfaces with cut loci containing compact subsets of infinite Hausdorff 1-measure? The example of a nontriangulable cut locus on the two-sphere constructed by Gluck and Singer [7] has finite Hausdorff 1-measure.

Several steps in the proof of Theorem 1 are valid in higher dimensions. Lemma 2.1 generalizes the main theorem in $[8]$ that the isometric immersion exists assuming $(*)$ when the cut locus and the conjugate locus of $M$ at $p$ coincide. Lemma 4.1 describes a condition intrinsic to $M$ under which Lemma 2.1 can be applied. However, we are presently able to verify the hypothesis of Lemma 4.1 for a somewhat general class of metrics only for surfaces, thus rendering Theorem 1.

1. Structure of the cut locus. Let $p$ be a point in a complete Riemannian manifold $M$ of dimension $n$, and let $C(p)$ be the cut locus of $p$ in $M$. A point $q \in C(p)$ is a conjugate cut point if $q$ is conjugate to $p$ along at least one minimizing geodesic joining $p$ to $q$ and is a nonconjugate cut point otherwise. The order of a nonconjugate cut point $q$ is defined to be the number (which is always finite and at least two) of minimizing geodesics joining $p$ to $q$. A nonconjugate cut point of order two will be called a cleave point for short.

PROPOSITION 1.1. The set of cleave points (or cleave locus) is relatively open in $C(p)$ and forms a smooth $(n-1)$-dimensional submanifold of $M$. The complement of the cleave locus in $C(p)$ is a closed subset of $M$ whose Hausdorff $(n-1)$-measure is zero.

PROOF. The first statement is a consequence of the local description of the nonconjugate cut locus contained in Propositions 2.3 and 2.4 of Ozols [14]. The complement of the cleave locus in $C(p)$ is thus closed in $M$, being a closed subset of the closed set $C(p)$. The Hausdorff $(n-1)$-measure of the conjugate cut locus is zero by the proofs of Proposition 2.2 in [9] and Lemma 1.1 in [17]. (See also the comments after Lemma 2.2 in [8].) By subadditivity of the Hausdorff $(n-1)$ measure, $H^{n-1}$, it remains to show $H^{n-1}(E)=0$ where $E$ is the set of nonconjugate cut points of order at least three.

To do this it is sufficient to show that each point of $E$ has a neighborhood in $M$ which intersects $E$ in a set of Hausdorff $(n-1)$-measure zero. Since $E$ can be covered by countably many such neighborhoods, it will follow that $H^{n-1}(E)=0$. 
Let $r \in E$. Ozols [14] constructs a neighborhood $U_{r}$ of $r$ in $M$ so that every minimizing geodesic from $p$ to a point of $U_{r}$ is close to (or associated to) exactly one of the minimizing geodesics from $p$ to $r$. Clearly, if $q \in U_{r}$, and there is a pair of minimizing geodesics joining $p$ to $q$ associated to the pair of minimizing geodesics from $p$ to $r$ indexed by $i$ and $j$, then $q \in K_{i j}^{r}$ where $K_{i j}^{r}$ is defined in [14]. Consequently every $q \in U_{r}$ for which there exist at least three minimizing geodesics joining $p$ to $q$ in $K_{i j}^{r} \cap K_{i k}^{r}$ for some three distinct indices $i, j, k$. Hence $E \cap U_{r} \subset \bigcup\left\{K_{i j}^{r} \cap K_{i k}^{r}: i, j, k\right.$ distinct $\}$. But $H^{n-1}\left(K_{i j}^{r} \cap K_{i k}^{r}\right)=0$ because $K_{i j}^{r} \cap K_{i k}^{r}$, being a transverse intersection of two smooth $(n-1)$-dimensional submanifolds by Propositions 2.4 and 2.6 of $[\mathbf{1 4}]$, is a smooth $(n-2)$-dimensional submanifold. Therefore $H^{n-1}\left(E \cap U_{r}\right)=0$.

2. The extension lemma. Throughout this section assume that $M$ and $\bar{M}$ are two complete, connected, $n$-dimensional Riemannian manifolds, that $I: T_{p} M \rightarrow$ $T_{\bar{p}} \bar{M}$ is a linear isometry, and that the condition (*) of the introduction holds. $M$ is not necessarily simply connected.

Let $\exp _{p}: T_{p} M \rightarrow M$ and $\exp _{\bar{p}}: T_{\bar{p}} \bar{M} \rightarrow \bar{M}$ denote the exponential maps. For every open set $V \subset T_{p} M$ for which the restriction $\exp _{p} \mid V$ is a diffeomorphism onto the open subset $\exp _{p}(V)$ of $M$, the proof of Lemma 1.35 of [4] shows that $\exp _{\bar{p}} \circ I \circ\left(\exp _{p} \mid V\right)^{-1}$ is an isometric immersion of $\exp _{p}(V)$ into $\bar{M}$. In particular, since $\exp _{p}$ restricted to $V_{p}=\left\{X \in T_{p} M\right.$ : $\exp _{p}(t X)$ contains no cut point for $0 \leq t \leq 1\}$ is well known to be a diffeomorphism onto the complement of $C(p)$ in $M, f=\exp _{\bar{p}} \circ I \circ\left(\exp _{p} \mid V_{p}\right)^{-1}: M-C(p) \rightarrow \bar{M}$ is an isometric immersion with $d f=I$ at $p$. Therefore, there exists a global isometric immersion $F: M \rightarrow \bar{M}$ with $d F=I$ at $p$ if and only if $f$ extends as an isometric immersion to all of $M$.

LEMMA 2.1. $f$ extends to an isometric immersion on all of $M$ if $\bar{g}_{1}(1)=\bar{g}_{2}(1)$ for every cleave point $q \in C(p)$ where $g_{1}, g_{2}:[0,1] \rightarrow M$ are the two minimizing geodesics joining $p$ to $q$ and $\bar{g}_{1}, \bar{g}_{2}$ the corresponding geodesics in $\bar{M}$.

(In other words, if $f$ extends continuously to the cleave locus, then it extends to all of $M$.

ProOF. Let $E$ be the set of cut points that are not cleave points. For $q \in M-E$, define $\bar{f}(q)=\bar{g}(1)$ where $\bar{g}$ is the geodesic in $\bar{M}$ that corresponds to any minimizing geodesic $g:[0,1] \rightarrow M$ joining $p$ to $q$. Clearly $\bar{f}$ extends $f$ to the open set $M-E$ and, by the condition of the lemma, is well defined. It will be shown that $\bar{f}$ is an isometric immersion. To do this it suffices to show $\bar{f}$ is an isometric immersion in a neighborhood of each cleave point because $\bar{f}$ agrees with the isometric immersion $f$ on $M-C(p)$.

Let $q \in C(p)$ be a cleave point, let $g_{1}$ and $g_{2}$ be the two minimizing geodesics joining $p$ to $q$, and let $X_{1}=g_{1}^{\prime}(0)$ and $X_{2}=g_{2}^{\prime}(0)$ be their initial tangent vectors. Thus $\exp _{p}\left(X_{1}\right)=\exp _{p}\left(X_{2}\right)=q$. Since $q$ is not conjugate to $p$ along $g_{1}$ or $g_{2}$, $\exp _{p}$ is nonsingular at $X_{1}$ and $X_{2}$. Thus there are connected neighborhoods $U_{q}$ of $q, V_{1}$ of $X_{1}$, and $V_{2}$ of $X_{2}$ so that $\exp _{p}$ carries both $V_{1}$ and $V_{2}$ diffeomorphically onto $U_{q}$. Thus as noted above the maps $f_{i}=\exp _{\bar{p}} \circ I \circ\left(\exp _{p} \mid V_{i}\right)^{-1}: U_{q} \rightarrow \bar{M}$ are isometric immersions for $i=1,2$. Consequently, $\bar{f}$ will be seen to be an isometric immersion in a neighborhood of $q$ once it is shown that $f_{1}=f_{2}=\bar{f}$ on $U_{q}$. 
In order to show $f_{1}=f_{2}$ on $U_{q}$ it is enough to show $d f_{1}=d f_{2}$ at $q$ because an isometric immersion between connected manifolds of the same dimension is determined by its differential at a point $[\mathbf{1 0}]$. Because the order of $q$ is two, $C(p) \cap$ $U_{q}=K$ is a smooth hypersurface in $U_{q}$ consisting entirely of cleave points and contains $q[\mathbf{1 4}]$. Clearly $f_{1}\left|K=f_{2}\right| K=\bar{f} \mid K$ by the assumption regarding cleave points. Hence the two linear isometries $d f_{1}, d f_{2}: T_{q} M \rightarrow T_{\bar{f}(q)} \bar{M}$ agree on the hyperplane $T_{q} K \subset T_{q}(M)$. Thus if $d f_{1} \neq d f_{2}$ at $q, d f_{1}=d f_{2} \circ R$ where $R: T_{q} M \rightarrow T_{q} M$ is reflection in the hyperplane $T_{q} K$. By Remark 2.5 of [14], both $g_{1}^{\prime}(1)$ and $g_{2}^{\prime}(1)$ make the same angle with every vector in $T_{q} K$. Hence $R\left(g_{1}^{\prime}(1)\right)=$ $g_{2}^{\prime}(1)$ because $g_{1}^{\prime}(1) \neq g_{2}^{\prime}(1)$ since the geodesics $g_{1}$ and $g_{2}$ are distinct. Also by construction of $f_{i}, d f_{i}\left(g_{i}^{\prime}(1)\right)=\bar{g}_{i}^{\prime}(1)$ where $\bar{g}_{i}$ is the geodesic in $\bar{M}$ corresponding to $g_{i}, i=1,2$. Thus, if $d f_{1} \neq d f_{2}$, then

$$
\bar{g}_{1}^{\prime}(1)=d f_{1}\left(g_{1}^{\prime}(1)\right)=d f_{2}\left(R\left(g_{1}^{\prime}(1)\right)\right)=d f_{2}\left(g_{2}^{\prime}(1)\right)=\bar{g}_{2}^{\prime}(1)
$$

This is a contradiction because $\bar{g}_{1} \neq \bar{g}_{2}$. Hence $d f_{1}=d f_{2}$ at $q$, and therefore $f_{1}=f_{2}$ on $U_{q}$.

Since $C(p) \cap U_{q}=K$, each point of $U_{q}-K$ is joined to $p$ by a unique minimizing geodesic, and this geodesic will be associated to exactly one of $g_{1}$ or $g_{2}$ as in the proof of Proposition 1.1, i.e. its initial tangent vector will be in either $V_{1}$ or $V_{2}$. Letting $U_{q}^{i}$ be the set of points in $U_{q}-K$ thus associated to $g_{i}$ for $i=1,2$, it follows that $f_{i}\left|U_{q}^{i}=\bar{f}\right| U_{q}^{i}$ by construction. Thus $\bar{f}=f_{1}=f_{2}$ on $U_{q}-K$ because $U_{q}-K=U_{q}^{1} \cup U_{q}^{2}$ and $f_{1}=f_{2}$ on $U_{q}$. Hence by continuity, $f_{1}=f_{2}=\bar{f}$ on $U_{q}$. Thus $\bar{f}: M-E \rightarrow \bar{M}$ is an isometric immersion.

Because $\bar{M}$ is complete, and $E$ is a closed set with $H^{n-1}(E)=0, \bar{f}$ extends to an isometric immersion defined on all of $M$. An outline of the proof of this fact follows; full details may be found in the proof of Theorem 3.1 in [8]. (1) Observe that the distance between two points in $M-E$ defined as the infimum of lengths of curves joining them in $M-E$ is the same as the distance between them in $M$. (This uses $H^{n-1}(E)=0$.) (2) $\bar{f}$ is a distance decreasing map since it preserves the lengths of curves, and hence extends to a distance decreasing map $F$ on $M$ because $M-E$ is dense in $M$ and $\bar{M}$ is complete. (3) Observe each point of $M$ has a neighborhood $U$ on which $F$ is distance increasing. (This uses $H^{n-1}(E)=0$ and strongly convex neighborhoods.) (4) Hence $F \mid U$ preserves the distance function and is therefore a Riemannian isometry by a theorem of Myers and Steenrod. Therefore $F: M \rightarrow \bar{M}$ is an isometric immersion.

3. Affine development. Several pertinent facts concerning the affine development of absolutely continuous curves in a Riemannian manifold $M$ are presented and summarized.

A continuous curve $u:[0,1] \rightarrow M$ is absolutely continuous if for every $\varepsilon>0$, there exists $\delta>0$ such that for every $0 \leq t_{1}<t_{2}<\cdots<t_{2 m} \leq 1$ with

$$
\sum_{i=1}^{m}\left|t_{2 i}-t_{2 i-1}\right|<\delta, \quad \sum_{i=1}^{m} \operatorname{dist}\left(u\left(t_{2 i}\right), u\left(t_{2 i-1}\right)\right)<\varepsilon .
$$

By a well-known theorem of Lebesgue, this is equivalent to the property that for every system of coordinates $\left(x_{1}, \ldots, x_{n}\right)$ on an open set $U$ in $M$, if $u([a, b]) \subset U$ 
where $[a, b] \subset[0,1]$, then the component functions $x_{i} \circ u$ of the curve are differentiable almost everywhere in $[a, b]$, the derivative functions $\left(x_{i} \circ u\right)^{\prime}(t)$ are Lebesgue integrable functions on $[a, b]$, and finally

$$
x_{i}(u(t))-x_{i}(u(a))=\int_{a}^{t}\left(x_{i} \circ u\right)^{\prime}(s) d s
$$

for all $t$ in $[a, b]$. In particular, an absolutely continuous curve $u$ in $M$ has a tangent vector $u^{\prime}(t) \in T_{u(t)} M$ for almost all $t$ in $[0,1]$.

Let $\mathrm{AC}(M)$ denote the set of all absolutely continuous curves in $M$ parameterized on the unit interval. A topology on $\mathrm{AC}(M)$ is obtained by taking as a subbase for the neighborhoods of $u \in \mathrm{AC}(M)$ the sets $N_{u}(U,[a, b], \varepsilon)$ defined as follows: let $x=\left(x_{1}, \ldots, x_{n}\right)$ be a coordinate system on the open set $U \subset M$ such that $u([a, b]) \subset U$, and let $\varepsilon>0$. Then $N_{u}(U,[a, b], \varepsilon)$ is the set of all $v \in \operatorname{AC}(M)$ such that $v([a, b]) \subset U$ and

$$
\left(\sup _{t \in[a, b]}|x(v(t))-x(u(t))|+\int_{a}^{b}\left|(x \circ v)^{\prime}(s)-(x \circ u)^{\prime}(s)\right| d s\right)<\varepsilon
$$

where || is the standard norm on $\mathbf{R}^{n}$. If $p \in M$, let $\mathrm{AC}_{p}(M)=\{u \in \mathrm{AC}(M): u(0)$ $=p\}$ be given the subspace topology.

The affine development of piecewise differentiable curves is explained in $\S 4$ of Chapter 3 in $[\mathbf{1 0}]$. The affine development of absolutely continuous curves may be defined by the same process. Let $u \in \mathrm{AC}_{p}(M)$. For each $t \in[0,1]$ where $u^{\prime}(t)$ is defined, parallel translate $u^{\prime}(t)$ along $u$ from $u(t)$ to $p$ to obtain a vector $v^{\prime}(t) \in T_{p} M . v^{\prime}$ is a Lebesgue integrable $T_{p} M$-valued function on $[0,1]$. Thus the curve $v(t)=\int_{0}^{t} v^{\prime}(s) d s$ is an absolutely continuous curve in $T_{p} M$ starting at the origin. By definition $\operatorname{Dev}_{p}(u)=v$. When $M$ is complete, each $v \in \mathrm{AC}_{0}\left(T_{p} M\right)$ is the development of a unique $u \in \mathrm{AC}_{p}(M)$. (See Theorem 4.1 on p. 172 of [10] for piecewise differentiable curves.) The above facts are elaborated upon in the appendix where the following theorem is proved.

THEOREM 3.1. Let $M$ be a complete Riemannian manifold, $p \in M$. Then $\operatorname{Dev}_{p}: \mathrm{AC}_{p}(M) \rightarrow \mathrm{AC}_{0}\left(T_{p} M\right)$ is a homeomorphism.

Because an isometry commutes with parallel translation, the following result is immediate.

Proposition 3.2. Let $M$ and $\bar{M}$ be two Riemannian manifolds of the same dimension, and let $U \subset M$ be open. If $f: U \rightarrow \bar{M}$ is an isometric immersion with $f(p)=\bar{p}$, then $d f_{p} \circ \operatorname{Dev}_{p}(u)=\operatorname{Dev}_{\bar{p}}(f \circ u)$ for all $u \in \operatorname{AC}_{p}(M)$ with $u([0,1]) \subset U$.

Let $\Gamma$ be a quotient space of the unit interval, and let $T:[0,1] \rightarrow \Gamma$ be the identification map. A continuous map $\bar{u}: \Gamma \rightarrow M$ (a manifold) is tree-formed in $M$ if (1) $u=\bar{u} \circ T$ is absolutely continuous, and (2) for every continuous 1-form $\varphi$ along $\bar{u}$ (i.e. a continuous map $\varphi: \Gamma \rightarrow T^{*} M$ such that $\varphi(x) \in T_{\bar{u}(x)}^{*} M$ for all $x \in \Gamma$ where $T^{*} M$ is the cotangent bundle),

$$
\int_{t_{0}}^{t_{1}} \varphi(T(s))\left(u^{\prime}(s)\right) d s=0
$$

whenever $T\left(t_{0}\right)=T\left(t_{1}\right)$. 
The notion of a tree-formed map is based upon that of a topological tree. If $\Gamma$ is a finite topological graph, and $\bar{u}: \Gamma \rightarrow M$ is a continuous map which is continuously differentiable when restricted to each edge, then every 1-form on $M$ can be pulled back to a "piecewise 1-form" on $\Gamma$. If $\Gamma$ is a tree, then this piecewise 1-form is exact. Hence its integral along every closed curve in $\Gamma$ is zero. Tree-formed maps allow certain nontriangulable sets to be considered.

The proof of the next result is deferred to the appendix.

THEOREM 3.3. Let $T:[0,1] \rightarrow \Gamma$ be an identification map. Let $M$ be a complete Riemannian manifold, and let $p \in M$.

(1) Suppose $\bar{u}: \Gamma \rightarrow M$ is tree-formed in $M, u=\bar{u} \circ T$, and $u(0)=p$. Then $v=\operatorname{Dev}_{p}(u)$ factors through $\Gamma$, and the induced map $\bar{v}: \Gamma \rightarrow T_{p} M$ is tree-formed in $T_{p} M$.

(2) Conversely, suppose $\bar{v}: \Gamma \rightarrow T_{p} M$ is tree-formed in $T_{p} M, v=\bar{v} \circ T$, and $v(0)=0$. Then $u=\operatorname{Dev}_{p}^{-1}(v)$ factors through $\Gamma$, and the induced map $\bar{u}: \Gamma \rightarrow M$ is tree-formed in $M$.

4. The hypothesis of the extension lemma. Resuming the assumptions of $\S 2$, let $f: M-C(p) \rightarrow \bar{M}$ denote the isometric immersion $f=\exp _{\bar{p}} \circ I \circ$ $\left(\exp _{p} \mid V_{p}\right)^{-1}$. The purpose of this section is to provide a condition which implies the hypothesis of Lemma 2.1, but depends only upon $M$.

LEMMA 4.1. Let $q \in C(p)$, and let $g_{1}, g_{2}:[0,1] \rightarrow M$ be two minimizing geodesics joining $p$ to $q$. Suppose that there exists an absolutely continuous curve $w:[0,1] \rightarrow M$ satisfying (i) $w(0)=w(1)=q$; (ii) $w=\bar{w} \circ T$ where $T:[0,1] \rightarrow \Gamma$ is an identification map with $T(0)=T(1)$, and $\bar{w}: \Gamma \rightarrow M$ is tree-formed in $M$; and (iii) there exists a sequence $w_{j} \in \mathrm{AC}(M)$ converging to $w$ in $\mathrm{AC}(M)$ such that $w_{j}(0)=g_{1}(1-1 / j), w_{j}(1)=g_{2}(1-1 / j)$, and $w_{j}([0,1]) \subset M-C(p)$ for all $j$. Then $\bar{g}_{1}(1)=\bar{g}_{2}(1)$ where $\bar{g}_{1}, \bar{g}_{2}$ are the geodesics in $\bar{M}$ corresponding to $g_{1}, g_{2}$.

PROOF. Define

$$
u_{j}(t)= \begin{cases}g_{1}(2 t(1-1 / j)) & \text { for } 0 \leq t \leq \frac{1}{2} \\ w_{j}(2 t-1) & \text { for } \frac{1}{2} \leq t \leq 1\end{cases}
$$

and

$$
u(t)= \begin{cases}g_{1}(2 t) & \text { for } 0 \leq t \leq \frac{1}{2} \\ w(2 t-1) & \text { for } \frac{1}{2} \leq t \leq 1\end{cases}
$$

Then $u_{j}$ converges to $u$ in $\operatorname{AC}_{p}(M)$. Set $v=\operatorname{Dev}_{\bar{p}}^{-1} \circ I \circ \operatorname{Dev}_{p}(u)$ and $v_{j}=$ $\operatorname{Dev}_{\bar{p}}^{-1} \circ I \circ \operatorname{Dev}_{p}\left(u_{j}\right)$. By Theorem 3.1, $v_{j}$ converges to $v$ in $\operatorname{AC}_{\bar{p}}(\bar{M})$. Since $u_{j}([0,1]) \subset M-C(p)$, and $f: M-C(p) \rightarrow \bar{M}$ is an isometric immersion, $v_{j}=f \circ u_{j}$ for all $j$ by Proposition 3.2. Thus $v_{j}\left(\frac{1}{2}\right)=\bar{g}_{1}(1-1 / j)$ and $v_{j}(1)=\bar{g}_{2}(1-1 / j)$ for all $j$. Hence, on taking limits, $v\left(\frac{1}{2}\right)=\bar{g}_{1}(1)$ and $v(1)=\bar{g}_{2}(1)$. It remains to prove $v\left(\frac{1}{2}\right)=v(1)$.

Let $\Gamma_{0}=[0,1] \cup \Gamma /\{1\} \sim\{T(0)\}$, i.e. $\Gamma_{0}$ is the identification space formed from the disjoint union of $[0,1]$ and $\Gamma$ by identifying the point $1 \in[0,1]$ with the point $T(0)=T(1) \in \Gamma$. The map $T_{0}:[0,1] \rightarrow \Gamma_{0}$ defined by

$$
T_{0}(t)= \begin{cases}2 t \in[0,1] & \text { for } 0 \leq t \leq \frac{1}{2} \\ T(2 t-1) & \text { for } \frac{1}{2} \leq t \leq 1\end{cases}
$$


is a quotient map. Clearly $u$ factors through $\Gamma_{0}$ and induces a map $\bar{u}: \Gamma_{0} \rightarrow M$ that equals $g_{1}$ on $[0,1] \subset \Gamma_{0}$ and equals $\bar{w}$ on $\Gamma \subset \Gamma_{0}$. Since $g_{1}$ and $\bar{w}$ are both treeformed in $M$, it is immediate from the construction that $\bar{u}$ is tree-formed in $M$. By Theorem 3.3, $v$ factors through $\Gamma_{0}$. Therefore $v\left(\frac{1}{2}\right)=v(1)$ because $T_{0}\left(\frac{1}{2}\right)=T_{0}(1)$. This completes the proof of the lemma.

The tangent cut locus is the set $C_{p}=\left\{X \in T_{p} M: \exp _{p}(X)\right.$ is the cut point along the geodesic $\left.\exp _{p}(t X)\right\}$.

LEMMA 4.2. Let $Y:[0,1] \rightarrow T_{p} M$ be an absolutely continuous curve with $Y([0,1]) \subset C_{p}$. Define geodesics $g_{1}(t)=\exp _{p}(t Y(0))$ and $g_{2}(t)=\exp _{p}(t Y(1))$ for $t \in[0,1]$. Set $w(t)=\exp _{p}(Y(t))$ and, for $0<c<1$, set $w_{c}(t)=\exp _{p}(c Y(t))$ for $t \in[0,1]$. Then the curves $w_{c}$ converge to $w$ in $\mathrm{AC}(M)$ as $c$ converges to 1 , and satisfy $w_{c}(0)=g_{1}(c), w_{c}(1)=g_{2}(c)$, and $w_{c}([0,1]) \subset M-C(p)$.

Proof. Clearly $c Y$ converges to $Y$ in $\operatorname{AC}\left(T_{p} M\right)$ as $c$ converges to 1 . Since $\exp _{p}$ is smooth, $\exp _{p}(c Y)$ converges to $\exp _{p}(Y)$ in $\operatorname{AC}(M)$. The other statements are obvious.

Consequently, a curve $w=\exp _{p} \circ Y$, as in Lemma 4.2, automatically satisfies condition (iii) in Lemma 4.1.

REMARKS. (1) If a cleave point $q$ satisfies the hypothesis of Lemma 4.1, then every point in the connected component of the cleave locus containing $q$ also satisfies the hypothesis. Because the cleave locus is a smooth codimension one submanifold, any $r$ in the same component as $q$ can be joined to $q$ by a smooth curve $u$. The curve made up of $u, w$, and $u^{-1}$ will satisfy conditions (i), (ii), and (iii) in Lemma 4.1 for $r$.

(2) There are obvious topological obstructions to the existence of the curve $w$ in Lemma 4.1. For example the loop made up of $g_{1}$ and $g_{2}^{-1}$ must be homologically trivial, and probably must be contractible. If the hypothesis of Lemma 4.1 is satisfied for all cleave points $q$, then $M$ must be simply connected because of Lemma 2.1 .

(3) If the cut locus of $M$ is $C^{1}$-triangulable, and it collapses simplicially in the sense of simple homotopy theory [5] to its $(n-2)$-skeleton, then the hypothesis of Lemma 4.1 is satisfied for every cleave point. This can be proved by induction on the number of elementary simplicial collapses needed to finally collapse the cell containing the given cleave point. There are however triangulable cut loci in simply connected manifolds that do not collapse simplicially to the $(n-2)$-skeleton. In simply-connected surfaces, triangulable cut loci are topological trees, and hence collapse simplicially to the zero-skeleton. By the methods of the next section, certain nontriangulable cut loci may be treated as well.

5. Surfaces. Throughout this section let $M$ be a complete, connected 2-dimensional Riemannian manifold. Fix $p \in M$. Introduce polar coordinates $(r, \theta) \in$ $[0, \infty) \times S_{p}$ in $T_{p} M$ where $S_{p}$ is the unit circle in $T_{p} M$. It is well known that the function $\mu: S_{p} \rightarrow[0, \infty]$ where $\mu(\theta)$ is the distance to the cut point (which is infinite if there is no cut point) along the ray defined by $\theta \in S_{p}$ is continuous. Moreover, by either $\left[\mathbf{1 2}\right.$ or 14], if the cut point along $\theta_{0}$ is a cleave point, then $\mu$ is smooth in a neighborhood of $\theta_{0}$ in $S_{p}$. Let $[\alpha, \beta]$ be an interval in the circle $S_{p}$ on which $\mu$ is finite. Then $Y(\theta)=(\mu(\theta), \theta)$ for $\theta \in[\alpha, \beta]$ defines a continuous curve in polar 
coordinates on $T_{p} M$ whose image is contained in the tangent cut locus $C_{p}$. Define $w(\theta)=\exp _{p}(Y(\theta))$ for $\theta \in[\alpha, \beta]$.

Proposition 5.1. The following are equivalent.

(i) $\mu$ is an absolutely continuous function on $[\alpha, \beta]$.

(ii) $Y:[\alpha, \beta] \rightarrow T_{p} M$ is absolutely continuous.

(iii) $w$ is an absolutely continuous curve in $M$.

(iv) The Hausdorff 1-measure of the image $w([\alpha, \beta]) \subset M$ is finite.

ProOF. The equivalence of (i) and (ii) is immediate from the definition of absolute continuity. Because $\exp _{p}$ is smooth, (ii) implies (iii). By the triangle inequality, $|\mu(\theta)-\mu(\bar{\theta})|=|\operatorname{dist}(p, w(\theta))-\operatorname{dist}(p, w(\bar{\theta}))| \leq \operatorname{dist}(w(\theta), w(\bar{\theta}))$. Therefore (iii) implies (i).

Now, for every $T \subset[\alpha, \beta]$ with Lebesgue measure zero, $H^{1}(w(T))=0$. To show this let $G=\{\theta \in[\alpha, \beta]: w(\theta)$ is a cleave point $\}$, and let $F=[\alpha, \beta]-G$. Then $G$ is open, and $\mu \mid G$ is smooth. Hence $w \mid G$ is smooth, and thus locally Lipschitzian. Consequently, $H^{1}(w(G \cap T))=0$ because $G \cap T$ has Lebesgue measure zero. On the other hand, $H^{1}(w(F \cap T)) \leq H^{1}(w(F))=0$ by Proposition 1.1. Therefore $H^{1}(w(T))=0$.

Thus Theorem 2.10.13 in Federer [6] applies, and one may conclude that $w$ is absolutely continuous if and only if

$$
V=\int_{M} N(w, y) d H^{1} y<\infty
$$

where $N(w, y)$ equals the number of elements in $w^{-1}(y) \subset[\alpha, \beta]$ for $y \in M$. (Of course $M$ is not a locally reflexive Banach space, but Remarks 3.2.46 in [6] apply.) Now $N(w, y)=0$ for $y \notin w([\alpha, \beta]), 1 \leq N(w, y) \leq 2$ for $y \in w(G)$, and $1 \leq$ $N(w, y)$ for $y \in w(F)$. Thus, because $H^{1}(w(F))=0$ and $w([\alpha, \beta])=w(G) \cup w(F)$, $H^{1}(w([\alpha, \beta])) \leq V \leq 2 \cdot H^{1}(w([\alpha, \beta]))$. Hence $V$ is finite if and only if $H^{1}(w([\alpha, \beta]))$ is finite. Therefore (iii) and (iv) are equivalent.

PROPOSITION 5.2. Let $g_{1}, g_{2}:[0,1] \rightarrow M$ be two minimizing geodesics joining $p$ to $q \in C(p)$. If $g_{1} \cup g_{2}$ bounds a disk $D$ in $M$, then $\mu$ is finite on the interval $[\alpha, \beta]=\left\{\theta \in S_{p}: \exp _{p}(t, \theta) \in D\right.$ for small positive $\left.t\right\}$. Furthermore, defining $w(\theta)=\exp _{p}(\mu(\theta), \theta)$ for $\theta \in[\alpha, \beta], \Gamma=w([\alpha, \beta])$, and $T:[\alpha, \beta] \rightarrow \Gamma$ by $T(\theta)=$ $w(\theta)$, the inclusion $\Gamma \subset M$ is tree-formed in $M$ provided $H^{1}(\Gamma)$ is finite.

PROOF. If there were no cut point on some geodesic of the form $\exp _{p}(t, \theta)$, $(t>0)$, for some $\theta \in[\alpha, \beta]$, then, since the diameter of the compact disk $D$ is finite, this geodesic would intersect the boundary of $D$ and thus intersect one of the minimizing geodesics $g_{1}$ or $g_{2}$. There would then exist a cut point along this geodesic. Therefore $\mu$ is finite on $[\alpha, \beta]$.

Because $T:[\alpha, \beta] \rightarrow \Gamma$ is a continuous surjective map between compact Hausdorff spaces, it is an identification map. Now suppose $H^{1}(\Gamma)$ is finite. By Proposition $5.1, w:[\alpha, \beta] \rightarrow M$ is absolutely continuous. Set $[\alpha, \beta]=F \cup G$ as in the proof of Proposition 5.1. Then the tangent vector $w^{\prime}(t)=0$ for almost all $t \in F$. This is because by Theorem 3.2.6 together with Remarks 3.2.46 in Federer [6],

$$
\int_{F}\left|w^{\prime}(s)\right| d s=\int_{M} N(w \mid F, y) d H^{1} y=0
$$


where $d s$ is Lebesgue measure on $[\alpha, \beta]$, and $N(w \mid F, y)$ is the number of points in $w^{-1}(y) \cap F$. The second integral is zero because $H^{1}(w(F))=0$, and $N(w \mid F, y)=0$ except for $y \in w(F)$. Also $w$ is smooth on $G$. In fact, writing $G \cap(\alpha, \beta)$ as a disjoint union of at most countably many intervals $I_{k}, w$ maps each $I_{k}$ diffeomorphically onto a connected component of the intersection $C^{*}$ of the cleave locus with the interior of the disk $D$. Since the boundary of $D$ is made up of two minimizing geodesics, the two minimizing geodesics joining $p$ to a point in $C^{*}$ must be contained in $D$. (See $\S 103$ of Blaschke [2].) Consequently, given a connected component of $C^{*}$, there are exactly two intervals, say $I_{k}$ and $I_{k^{\prime}}$, which map diffeomorphically onto the given component, and furthermore $w \mid I_{k}$ and $w \mid I_{k^{\prime}}$ traverse the component in opposite directions.

Let $\varphi: \Gamma \rightarrow T^{*} M$ be a continuous 1 -form along $\Gamma$. Set

$$
a_{k}=\int_{I_{k}} \varphi(w(s))\left(w^{\prime}(s)\right) d s .
$$

Then

$$
\begin{aligned}
\sum_{k}\left|a_{k}\right| & \leq \sum_{k} \int_{I_{k}}\left|\varphi(w(s))\left(w^{\prime}(s)\right)\right| d s \\
& =\int_{G}\left|\varphi(w(s))\left(w^{\prime}(s)\right)\right| d s<\infty
\end{aligned}
$$

shows $\sum_{k} a_{k}$ is absolutely convergent. Since $w \mid I_{k}$ and $w \mid I_{k^{\prime}}$ traverse the same curve in opposite directions, $a_{k}=-a_{k^{\prime}}$. Thus by rearranging terms, $\sum_{k} a_{k}=0$. Now

$$
\begin{aligned}
\int_{\alpha}^{\beta} \varphi(T(s))\left(w^{\prime}(s)\right) d s & =\int_{G} \varphi(T(s))\left(w^{\prime}(s)\right) d s+\int_{F} \varphi(T(s))\left(w^{\prime}(s)\right) d s \\
& =\int_{G} \varphi(T(s))\left(w^{\prime}(s)\right) d s=\sum_{k} a_{k}=0
\end{aligned}
$$

because $w^{\prime}(s)=0$ for almost all $s$ in $F$. Similar considerations show

$$
\int_{t_{0}}^{t_{1}} \varphi\left(T(s)\left(w^{\prime}(s)\right) d s=0\right.
$$

whenever $T\left(t_{0}\right)=T\left(t_{1}\right)$. Therefore $\Gamma$ is tree-formed in $M$.

The proof of Theorem 1 can now be given. If $M$ is a simply connected surface, and $q$ is a cleave point, then the two minimizing geodesics joining $p$ to $q$ bound a disk in $M$. If every compact subset of $C(p)$ has finite Hausdorff 1-measure, then the isometric immersion $F: M \rightarrow \bar{M}$ exists by Lemmas 2.1, 4.1, 4.2, and Proposition 5.2 .

6. Appendix - An integral equation. The topology on $\operatorname{AC}\left(R^{n}\right)$ is induced by the norm $\|u\|=\|u\|_{\infty}+\left\|u^{\prime}\right\|_{1}$ where $\|u\|_{\infty}=\sup \{|u(s)|: s \in[0,1]\},\left\|u^{\prime}\right\|_{1}=$ $\int_{0}^{1}\left|u^{\prime}(s)\right| d s$, and || is a norm on $\mathbf{R}^{n}$.

Let $A: \mathbf{R}^{n} \times \mathbf{R}^{m} \rightarrow \mathbf{R}_{n}^{m}$ be a continuous function where $\mathbf{R}_{n}^{m}$ is the vector space of $m \times n$ matrices. Assume $A$ satisfies the Lipschitz condition

$$
|A(x, y)-A(x, \bar{y})| \leq h(x)|y-\bar{y}|
$$

for all $x \in \mathbf{R}^{n}$ and $y, \bar{y} \in \mathbf{R}^{m}$ where $h: \mathbf{R}^{m} \rightarrow \mathbf{R}$ is continuous. Fix $p \in \mathbf{R}^{n}$ and $q \in \mathbf{R}^{m}$. 
Proposition 6.1. For every $u \in \mathrm{AC}_{p}\left(\mathbf{R}^{n}\right)$, the integral equation

$$
v(t)=q+\int_{0}^{t} A(u(s), v(s)) u^{\prime}(s) d s, \quad t \in[0,1],
$$

has a unique solution. The resulting mapping $\mathrm{AC}_{p}\left(\mathbf{R}^{n}\right) \rightarrow \mathrm{AC}_{q}\left(\mathbf{R}^{m}\right)$ which sends $u$ to the solution $v$ is continuous.

PROOF. The kernel $k_{u}(s, z)=A(u(s), z) u^{\prime}(s)$ satisfies the generalized Lipschitz condition $\left|k_{u}(s, z)-k_{u}(s, \bar{z})\right| \leq l(s)|z-\bar{z}|$ where $l(s)=|h(u(s))|\left|u^{\prime}(s)\right|$ is Lebesgue integrable on $[0,1]$. Hence the integral equation has a unique solution which may be obtained as the uniform limit of successive approximations (pp. 61-62 of [16]).

To prove continuity let $u, \bar{u} \in \mathrm{AC}_{p}\left(\mathbf{R}^{n}\right)$, and let $v, \bar{v} \in \mathrm{AC}_{q}\left(\mathbf{R}^{m}\right)$ be the respective solutions. Then for almost all $s$, where we leave out $s$ for greater readability,

$$
\begin{aligned}
\left|A(\bar{u}, \bar{v}) \bar{u}^{\prime}-A(u, v) u^{\prime}\right| \leq & |A(\bar{u}, v)-A(u, v)|\left|u^{\prime}\right|+|A(\bar{u}, v)|\left|\bar{u}^{\prime}-u^{\prime}\right| \\
& +|A(\bar{u}, \bar{v})-A(\bar{u}, v)|\left(\left|\bar{u}^{\prime}-u^{\prime}\right|+\left|u^{\prime}\right|\right) \\
\leq & g_{\bar{u} u}(s)+h_{\bar{u} u}(s) \bar{v}(s)-v(s)
\end{aligned}
$$

where

$$
g_{\bar{u} u}(s)=|A(\bar{u}, v)-A(u, v)|\left|u^{\prime}\right|+|A(\bar{u}, v)|\left|\bar{u}^{\prime}-u^{\prime}\right|
$$

and

$$
h_{\bar{u} u}(s)=|h(\bar{u})|\left(\left|\bar{u}^{\prime}-u^{\prime}\right|+\left|u^{\prime}\right|\right) .
$$

Set $G_{\bar{u} u}=\int_{0}^{1} g_{\bar{u} u}(s) d s$ and $H_{\bar{u} u}(t)=\int_{0}^{t} h_{\bar{u} u}(s) d s$ for $t \in[0,1]$. Then for fixed $u$, $G_{\bar{u} u} \rightarrow 0$ and $H_{\bar{u} u}(t)$ stays bounded as $\bar{u} \rightarrow u$. By (1) and (2),

$$
\begin{aligned}
|\bar{v}(t)-v(t)| & \leq \int_{0}^{t}\left|A(\bar{u}, \bar{v}) \bar{u}^{\prime}-A(u, v) u^{\prime}\right| d s \\
& \leq G_{\bar{u} u}+\int_{0}^{t} h_{\bar{u} u}(s)|\bar{v}(s)-v(s)| d s .
\end{aligned}
$$

Hence by Gronwall's inequality [16] and (3)

$$
|\bar{v}(t)-v(t)| \leq G_{\bar{u} u} \exp \left(H_{\bar{u} u}(t)\right) .
$$

Thus for fixed $u,\|\bar{v}-v\|_{\infty} \rightarrow 0$ as $\bar{u} \rightarrow u$. Also by (1), (2), and (4),

$$
\begin{aligned}
\left\|\bar{v}^{\prime}-v^{\prime}\right\|_{1} & =\int_{0}^{1}\left|A(\bar{u}, \bar{v}) \bar{u}^{\prime}-A(u, v) u^{\prime}\right| d s \\
& \leq \int_{0}^{1} g_{\bar{u} u}(s)+h_{\bar{u} u}(s) G_{\bar{u} u} \exp \left(H_{\bar{u} u}(s)\right) d s \\
& \leq G_{\bar{u} u}\left(1+\exp \left(2 H_{\bar{u} u}(1)\right) .\right.
\end{aligned}
$$

Thus $\left\|\bar{v}^{\prime}-v^{\prime}\right\|_{1} \rightarrow 0$ as $\bar{u} \rightarrow u$ for fixed $u$. Therefore $\|\bar{v}-v\| \rightarrow 0$ as $\bar{u} \rightarrow u$ for fixed $u$.

Given an identification map $T:[0,1] \rightarrow \Gamma$, a continuous map $\bar{u}: \Gamma \rightarrow \mathbf{R}^{n}$ will be tree-formed in $\mathbf{R}^{n}$ if (1) $u=\bar{u} \circ T$ is absolutely continuous, and (2) for every continuous map $\varphi: \Gamma \rightarrow\left(\mathbf{R}^{n}\right)^{*}$, the dual space of $\mathbf{R}^{n}$ which we identify with the space of $1 \times n$ matrices,

$$
\int_{t_{0}}^{t_{1}} \varphi(T(s)) u^{\prime}(s) d s=0
$$


whenever $T\left(t_{0}\right)=T\left(t_{1}\right)$. Consequently, if $\bar{u}$ is tree-formed, and $\Omega: \Gamma \rightarrow \mathbf{R}_{m}^{n}$ is continuous, then

$$
\int_{t_{0}}^{t_{1}} \Omega(T(s)) u^{\prime}(s) d s=0
$$

whenever $T\left(t_{0}\right)=T\left(t_{1}\right)$ since one may integrate each component of the vectorvalued integral separately.

Proposition 6.2. Let $T:[0,1] \rightarrow \Gamma$ be a quotient map. Suppose $\bar{u}: \Gamma \rightarrow \mathbf{R}^{n}$ is tree-formed in $\mathbf{R}^{n}$, and $u=\bar{u} \circ T \in \mathrm{AC}_{p}\left(\mathbf{R}^{n}\right)$. Then the solution $v$ of (1) factors through $\Gamma$, and the induced map $\bar{v}: \Gamma \rightarrow \mathbf{R}^{m}$ is tree-formed in $\mathbf{R}^{m}$.

ProOF. Define the successive approximations: $v_{0}(t)=q, v_{i+1}(t)=q+$ $\int_{0}^{t} A\left(u(s), v_{i}(s)\right) u^{\prime}(s) d s$ for $t \in[0,1]$. Then $v_{i}$ factors through $\Gamma$ for all $i$. The proof is by induction on $i$. Trivially, $v_{0}=\bar{v}_{0} \circ T$ where $\bar{v}_{0}=q$. Assume $\bar{v}_{i}: \Gamma \rightarrow \mathbf{R}^{m}$ is continuous, and $v_{i}=\bar{v}_{i} \circ T$. Define the continuous map $\Omega: \Gamma \rightarrow \mathbf{R}_{n}^{m}$ by $\Omega(-)=A\left(\bar{u}(-), \bar{v}_{i}(-)\right)$. If $T\left(t_{0}\right)=T\left(t_{1}\right)$, then

$$
\begin{aligned}
v_{i+1}\left(t_{1}\right)-v_{i+1}\left(t_{0}\right) & =\int_{t_{0}}^{t_{1}} A\left(u(s), v_{i}(s)\right) u^{\prime}(s) d s \\
& =\int_{t_{0}}^{t_{1}} \Omega(T(s)) u^{\prime}(s) d s=0
\end{aligned}
$$

since $\bar{u} \circ T=u, \bar{v}_{i} \circ T=v_{i}$, and $\bar{u}$ is tree-formed in $\mathbf{R}^{n}$. Hence $v_{i+1}$ induces a continuous map $\bar{v}_{i+1}: \Gamma \rightarrow \mathbf{R}^{m}$ such that $v_{i+1}=\bar{v}_{i+1} \circ T$.

As remarked in the proof of Proposition 6.1, the successive approximations $v_{i}$ converge to $v$ uniformly. Thus if $T\left(t_{0}\right)=T\left(t_{1}\right)$, then $v_{i}\left(t_{0}\right)=v_{i}\left(t_{1}\right)$ for all $i$, and on taking limits $v\left(t_{0}\right)=v\left(t_{1}\right)$. This shows $v$ induces a continuous map $\bar{v}: \Gamma \rightarrow \mathbf{R}^{m}$ satisfying $v=\bar{v} \circ T$. It remains to show that $\bar{v}$ is tree-formed in $\mathbf{R}^{m}$.

Since $v$ is the solution of equation (1), it is absolutely continuous, and $v^{\prime}(s)=$ $A(u(s), v(s)) u^{\prime}(s)$ for almost all $s$. Let $\varphi: \Gamma \rightarrow\left(\mathbf{R}^{m}\right)^{*}$ be continuous, and define the continuous map $\bar{\varphi}: \Gamma \rightarrow\left(\mathbf{R}^{n}\right)^{*}$ by $\bar{\varphi}(-)=\varphi(-) A(\bar{u}(-), \bar{v}(-))$. Then

$$
\begin{aligned}
\int_{t_{0}}^{t_{1}} \varphi(T(s)) v^{\prime}(s) d s & =\int_{t_{0}}^{t_{1}} \varphi(T(s)) A(u(s), v(s)) u^{\prime}(s) d s \\
& =\int_{t_{0}}^{t_{1}} \bar{\varphi}(T(s)) u^{\prime}(s) d s=0
\end{aligned}
$$

if $T\left(t_{0}\right)=T\left(t_{1}\right)$ because $\bar{u}$ is tree-formed in $\mathbf{R}^{n}$. Thus $\bar{v}$ is tree-formed in $\mathbf{R}^{m}$.

In order to obtain Theorems 3.1 and 3.3 as special cases of Propositions 6.1 and 6.2 , assume $i: M \rightarrow \mathbf{R}^{N}$ is an isometric embedding of the complete $n$-dimensional Riemannian manifold $M$ into some Euclidean space [13]. A continuous curve $u$ in $M$ is absolutely continuous if and only if $i \circ u$ is absolutely continuous in $\mathbf{R}^{N}$. In this way $\mathrm{AC}(M)$ is a subspace of $\operatorname{AC}\left(\mathbf{R}^{N}\right)$. Also a map $\bar{u}: \Gamma \rightarrow M$ is tree-formed in $M$ if and only if $i \circ \bar{u}$ is tree-formed in $\mathbf{R}^{N}$.

For each $x \in M, T_{x} M$ is considered to be an $n$-dimensional vector subspace of $\mathbf{R}^{N}$ in the usual way. Let $P(x): \mathbf{R}^{N} \rightarrow \mathbf{R}^{N}$ be the orthogonal projection operator of $\mathbf{R}^{N}$ corresponding to $T_{x} M$. Thus $P(x)$ is an $N \times N$ matrix, and a vector $X \in \mathbf{R}^{N}$ is tangent (respectively perpendicular) to $M$ at $x$ if and only if $P(x) X=X$ 
(respectively $P(x) X=0$ ). The mapping $P: M \rightarrow \mathbf{R}_{N}^{N}$ is smooth. Extend $P$ in any way to a smooth mapping $P: \mathbf{R}^{N} \rightarrow \mathbf{R}_{N}^{N}$.

Define $A: \mathbf{R}^{N} \times \mathbf{R}_{n}^{N} \rightarrow \mathbf{R}_{N}^{N n}$ where $\mathbf{R}_{N}^{N n}$ denotes $N$-tuples of $N \times n$ matrices by the formula

$$
A(x, y)=\left(\frac{\partial P}{\partial x_{1}}(x) y, \ldots, \frac{\partial P}{\partial x_{N}}(x) y\right)
$$

for $x \in \mathbf{R}^{N}, y \in \mathbf{R}_{n}^{N}$. Clearly $A$ satisfies the above Lipschitz condition with

$$
h(x)=\max \left\{\left|\frac{\partial P}{\partial x_{j}}(x)\right|: j=1, \ldots, N\right\} .
$$

Fix a point $p$ in $M$ and an orthonormal basis $q$ of $T_{p} M$ so that $q \in \mathbf{R}_{n}^{N}$.

LEMMA 6.3. If $A, p, q$ are as just defined, and $u \in \mathrm{AC}_{p}(M)$, then the solution of the integral equation (1) is a parallel orthonormal frame along $u$.

Proof. Let $u=\left(u_{1}, \ldots, u_{N}\right)$ be the components of $u$. Then the differential equation corresponding to (1) can be expressed:

$$
v^{\prime}(t)=\sum_{j=1}^{N} \frac{\partial P}{\partial x_{j}}(u(t)) v(t) u_{j}^{\prime}(t)=(P(u(t)))^{\prime} v(t)
$$

with initial condition $v(0)=q$. This is equivalent to the pair of equations:

$$
P(u(t)) v(t)=v(t) \quad \text { and } \quad P(u(t)) v^{\prime}(t)=0 \quad \text { with } v(0)=q .
$$

(The equivalence may be proved by differentiating (6).) The first equation in (6) shows $v$ is tangent along $u$ while the second that $v$ is parallel along $u$ (its derivative is perpendicular to $M$ ).

Given $u$ and $v$ in Lemma 6.3, by definition of parallel translation and development, observe that $v(t): \mathbf{R}^{n} \rightarrow T_{u(t)} M$ is a linear isometry for all $t$, and that

$$
w(t)=\int_{0}^{t}(v(s))^{-1} u^{\prime}(s) d s
$$

is the absolutely continuous curve in $\mathbf{R}^{n}$ starting at 0 such that $q \circ w=\operatorname{Dev}_{p}(u)$ where $q: \mathbf{R}^{n} \rightarrow T_{p} M$ is considered to be a linear isometry. Clearly because $v$ depends continuously on $u \in \mathrm{AC}_{p}(M)$ by Proposition $6.1, w$ does as well. Thus $\operatorname{Dev}_{p}$ is continuous. If $u=\bar{u} \circ T$ where $T:[0,1] \rightarrow \Gamma$ is an identification map and $\bar{u}: \Gamma \rightarrow M$ is tree-formed, then by Proposition 6.2 there is a continuous map $\bar{v}: \Gamma \rightarrow \mathbf{R}_{n}^{N}$ such that $v=\bar{v} \circ T$. Hence if $\varphi: \Gamma \rightarrow\left(\mathbf{R}^{n}\right)^{*}$ is continuous, then $\bar{\varphi}(-)=\varphi(-)(\bar{v}(-))^{-1}$ is a continuous 1 -form along $\bar{u}$. Therefore

$$
\begin{aligned}
\int_{t_{0}}^{t_{1}} \varphi(T(s)) w^{\prime}(s) d s & =\int_{t_{0}}^{t_{1}} \varphi(T(s))(v(s))^{-1} u^{\prime}(s) d s \\
& =\int_{t_{0}}^{t_{1}} \bar{\varphi}(T(s)) u^{\prime}(s) d s=0
\end{aligned}
$$

if $T\left(t_{0}\right)=T\left(t_{1}\right)$ because $\bar{u}$ is tree-formed. Thus $w$ factors through $\Gamma$ inducing a tree-formed map $\bar{w}: \Gamma \rightarrow \mathbf{R}^{n}$. Since $\operatorname{Dev}_{p}(u)=q \circ w$, the first part of Theorem 3.3 is proved. 
In order to study the inverse of development, define $A: \mathbf{R}^{n} \times\left(\mathbf{R}^{N} \times \mathbf{R}_{n}^{N}\right) \rightarrow \mathbf{R}_{n}^{N} \times$ $\mathbf{R}_{n}^{N n}$ where $\mathbf{R}_{n}^{N n}$ are $n$-tuples of $N \times n$ matrices, by $A(w,(x, y))=\left(A_{1}(w,(x, y))\right.$, $\left.A_{2}(w,(x, y))\right)$ where $A_{1}(w,(x, y))=y$ and

$$
A_{2}(w,(x, y))=\left(\sum_{j=1}^{N} \frac{\partial P}{\partial x_{j}}(x) y_{1}^{j} y, \ldots, \sum_{j=1}^{N} \frac{\partial P}{\partial x_{j}}(x) y_{n}^{j} y\right) .
$$

(Here $y_{i}^{j}$ are the components of the $N \times n$ matrix $y$.) Fix $p \in M$, and an orthonormal basis $q$ of $T_{p} M$ so that $q \in \mathbf{R}_{n}^{N}$.

LEMma 6.4. For $w \in \mathrm{AC}_{0}\left(\mathbf{R}^{n}\right)$, let $(u, v) \in \mathrm{AC}_{(p, q)}\left(\mathbf{R}_{n}^{N} \times \mathbf{R}_{n}^{N n}\right)$ satisfy the integral equation

$$
(u(t), v(t))=(p, q)+\int_{0}^{t} A(w(s),(u(s), v(s))) w^{\prime}(s) d s .
$$

Then $q \circ w$ is the development of $u$.

Proof. Using the form of $A_{1}$ and $A_{2}$, the differential equations take the form:

$$
u^{\prime}(t)=v(t) w^{\prime}(t) \quad \text { and } \quad v^{\prime}(t)=\sum_{i=1}^{n} \sum_{j=1}^{N} \frac{\partial P}{\partial x_{j}}(u(t)) v_{i}^{j}(t) w_{i}^{\prime}(t) v(t) .
$$

By the first equation and the definition of development it suffices to show $v$ is parallel along $u$. But by the first equation $u_{j}^{\prime}(t)=\sum_{i=1}^{n} v_{i}^{j}(t) w_{i}^{\prime}(t)$ which when substituted into the second gives (5) from Lemma 6.3. Hence $v$ is parallel along $u$ and the lemma is proved. (The completeness of $M$ is needed to conclude $u(t)$ never leaves $M$ as in the proof of Theorem 4.1 on p. 172 of $[\mathbf{1 0}]$.)

Existence and uniqueness for (7) would show $\operatorname{Dev}_{p}: \mathrm{AC}_{p}(M) \rightarrow \mathrm{AC}_{0}\left(T_{p} M\right)$ is one-one and onto. Unfortunately, Propositions 6.1 and 6.2 cannot be immediately applied because $A$ does not satisfy the Lipschitz condition because it depends quadratically on $y$. However, for every $C>0$, define $A_{C}(w,(x, y))=A\left(w,(x, y)_{C}\right)$ where

$$
(x, y)_{C}= \begin{cases}(x, y) & \text { if }|(x, y)| \leq C, \\ (x, y) /|(x, y)| & \text { if }|(x, y)| \geq C,\end{cases}
$$

then obviously $A_{C}$ satisfies the Lipschitz condition and any solution $(u, v)$ of $(7)$ with $A$ replaced by $A_{C}$ is a solution of $(7)$ provided $|(u(t), v(t))|<C$ for all $t$. Now if $(u, v)$ is a solution of $(7)$, then $v(t)$ is an orthonormal frame, and hence stays in a bounded set of $\mathbf{R}_{n}^{N}$. Also the length of $u$ equals the length of $w$. Thus for each $L>0$, there exists a $C>0$ such that the solution $(u, v)$ of $(7)$ corresponding to $w \in D_{L}=$ $\left\{w \in \mathrm{AC}_{0}\left(\mathbf{R}^{n}\right):\|w\|<L\right\}$ stays bounded by $C$. Hence, by applying Propositions 6.1 and 6.2 with $A_{C}$, it follows that $u$ is unique and depends continuously on $w$ and will factor through a tree-formed map if $w$ does provided $w \in D_{L}$. Because $\mathrm{AC}_{0}\left(\mathbf{R}^{n}\right)=\bigcup_{L>0} D_{L}$, the proofs of Theorems 3.1 and 3.3 are complete.

\section{REFERENCES}

1. W. Ambrose, Parallel translation of Riemannian curvature, Ann. of Math. 64 (1956), 337-363.

2. W. Blaschke, Differential geometry. I, Chelsea, New York, 1967. 
3. M. Buchner, Simplicial structure of the real analytic cut locus, Proc. Amer. Math. Soc. 66 (1977), 118-121.

4. J. Cheeger and D. Ebin, Comparison theorems in Riemannian geometry, North-Holland, Amsterdam, 1975.

5. M. M. Cohen, A course in simple-homotopy theory, Springer-Verlag, Berlin and New York, 1973.

6. H. Federer, Geometric measure theory, Springer-Verlag, Berlin and New York, 1969.

7. H. Gluck and D. Singer, Scattering of geodesic fields. I, Ann. of Math. (2) 108 (1978), 347-372.

8. J. Hebda, Conjugate and cut loci and the Cartan-Ambrose-Hicks theorem, Indiana Univ. Math. J. 31 (1982), 17-25.

9. (1983), 45-52.

10. S. Kobayashi and K. Nomizu, Foundations of differential geometry. I, Interscience, New York and London, 1963.

11. S. B. Myers, Riemannian manifolds in the large, Duke Math. J. 1 (1935), 39-49.

12. __ Connections between differential geometry and topology. I, II, Duke Math. J. 1 (1935), 376-391; ibid. 2 (1936), 95-102.

13. J. F. Nash, The imbedding problem for Riemannian manifolds, Ann. of Math. (2) 63 (1956), 20-63.

14. V. Ozols, Cut loci in Riemannian manifolds, Tôhoku Math. J. 26 (1974), 219-227.

15. C. T. C. Wall, Geometric properties of generic differential manifolds, Geometry and Topology, Lecture Notes in Math., vol. 597, Springer-Verlag, Berlin and New York, 1977.

16. W. Walter, Differential and integral inequalities, Springer-Verlag, Berlin and New York, 1970.

17. F. W. Warner, Conjugate loci of constant order, Ann. of Math. (2) 86 (1967), 192-212.

Department of Mathematics and Computer Science, St. Louis University, ST. LOUIS, MISSOURI 63103 